Für eine wirksame Tätigkeit des SFV im Bereich der Fremdenverkehrspolitik unerläßlich ist sodann die Ausrichtung der Fremdenverkehrswirtschaft auf ein gemeinsames Ziel.

Die Interessenwahrung und Mitsprache der Tourismusträger bei allen fremdenverkehrspolitischen Entscheidungen ist eine weitere wichtige Aufgabe des SFV. Im Verlaufe des Gesetzgebungsverfahrens spielen - neben den Gesprächen in den parlamentarischen Vorzimmern sowie der Einsitznahme von Verbandsexponenten in der Legislative - vor allem die Stellungnahmen im Rahmen der Vernehmlassungsverfahren eine zentrale Rolle. In aufwendiger Arbeit werden in dieser Phase Grundsatzüberlegungen zu kommenden Gesetzesvorlagen angestellt und vorausschauend die sich für den Tourismus ergebenden Konsequenzen beurteilt und öffentlich dargelegt. Dabei werden die sich stellenden Fragen gemeinsam mit den wichtigsten im Tourismus tätigen Institutionen angegangen, insbesondere mit der Schweizerischen Verkehrszentrale, dem Schweizer Hotelier-Verein, dem Schweizer Wirteverband sowie dem Verband Schweizerischer Kur- und Verkehrsdirektoren. Unter der Federführung des SFV wurde so in den letzten fünf Jahren zu 20 Bundesgesetzen oder Verordnungen eine Vernehmlassung eingereicht, u. a. zu den Richtlinien betreffend Eingriffe in die Landschaft im Interesse des Skisportes, zur Verordnung über die Konzessionierung von Luftseilbahnen, zum Umweltschutzgesetz, zur Verordnung über die Benützung des eidg. Kartenwerkes und der Pläne der Grundbuchvermessung, zur Gesamtverkehrs- und zur Gesamtenergiekonzeption, zur Lex Furgler sowie auch zum Fußund Wanderweggesetz. Die wichtige Funktion der Mitsprache erfüllt der SFV sodann auch auf interna- tionaler Ebene, nämlich als Mitglied der Schweizer Delegation im OECD-Tourismuskomitee.

Als dritter wesentlicher Aufgabenbereich obliegt dem SFV die Informations- und Beratungstätigkeit. $\mathrm{Zu}$ diesem Zweck ist dem Verband eine Dokumentationsund Beratungsstelle angegliedert, die sich vor allem mit der Erarbeitung von Grundlagen zu einer langfristigen Verbesserung des touristischen Angebotes der Schweiz befaßt. So werden im In- und Ausland umfassende Informationen und Erfahrungen gesammelt und in einschlägigen Berichten ausgewertet. In jüngster Vergangenheit wurden Themen wie «Sportanlagen in Ferienorten", "Die Kurtaxe - eine taugliche Finanzquelle?» oder "Aparthotels - Entwicklung der Hotellerie auf neuen Wegen?» abgehandelt, Probleme bezüglich des Verkehrs in Naherholungsgebieten oder hinsichtlich des Campingtourismus untersucht sowie Leitbilder für verschiedene Fremdenverkehrsorte und -regionen erstellt. An die Kosten dieser gemeinwohlorientierten Tätigkeit leistet der Bund einen jährlichen Beitrag von 100000 Franken.

Nicht zuletzt möchte der SFV mit gezielter Öffentlichkeitsarbeit über die Bedeutung des Tourismus informieren und zu einem vermehrten Tourismusbewußtsein in der Bevölkerung beitragen. Zu diesem Zweck gibt er u.a. ein vierteljährlich erscheinendes Bulletin heraus. Mit seinen Informationen will der SFV indessen nicht ein rosarotes Bild der Fremdenverkehrswirtschaft malen, sondern er bemüht sich, Probleme und Lösungsmöglichkeiten stets offen darzulegen.

Schweizerischer Fremdenverkehrsverband (SFV)

Monbijoustr. 130, 3001 Bern, Tel. 031/45 8745

\title{
Das Forschungsinstitut für Fremdenverkehr der Universität Bern
}

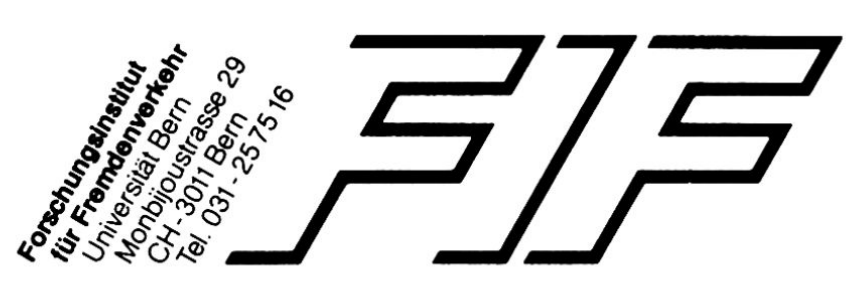

Mitten in den politischen und wirtschaftlichen Krisenjahren wurde 1941 an der Universität Bern das Forschungsinstitut für Fremdenverkehr (FIF) gegründet. Den damaligen Initianten darf ein hohes $\mathrm{Ma} B$ an Mut und Weitblick attestiert werden, war doch seinerzeit der Fremdenverkehr noch kaum ein hochschulfähiges Nebenfach. Die Leitung des Instituts war zu Beginn dem Inhaber des ordentlichen Lehrstuhls für Betriebswirtschaft (Prof. A. Walther) nebenamtlich übertragen. Aufgrund dieser Doppelfunktion konnte er nur zirka einēn Fünftel seiner Tätigkeit zugunsten 
des FIF einsetzen. Insgesamt arbeiteten in den ersten Jahren bloß $13 / 4$ Arbeitskräfte (der Direktor eingerechnet) am Institut.

Unter der zielstrebigen Leitung so bekannter Persönlichkeiten wie der Professoren Kurt Krapf (1943-1963), Paul Risch (1963-1971) und Jost Krippendorf (seit 1971) entwickelte sich das FIF aus den bescheidenen Anfängen zu einem international bekannten und auf dem Gebiet des Fremdenverkehrs führenden Institut, an dem heute insgesamt acht Angestellte arbeiten. $\mathrm{Da} B$ sich in den vierzig Jahren seit Bestehen des FIF nicht nur personell einiges verändert hat, mag die folgende kurze Beschreibung der vier heute gültigen Institutszwecke nach dem Kriterium «einst und jetzt» anschaulich aufzeigen.

\section{Forschung}

Grundpfeiler jeder Tätigkeit eines Universitätsinstituts bildet die Forschung. Aus naheliegenden Gründen war diese in den vierziger Jahren betont betriebswirtschaftlich orientiert (insbesondere Organisation und Rechnungswesen im Gastgewerbe). Nach und nach wurde das Forschungsfeld immer mehr ausgeweitet: Standen später eher volkswirtschaftliche Fragen sowie Planungsprobleme in Kurorten und Regionen im Vordergrund, so liegt zur Zeit das Schwergewicht eindeutig auf dem Gesellschafts- und Umweltbereich. Zusätzlich wird der Fremdenverkehr in das größere Umfeld der Freizeit gestellt (Tourismus als eine der vielen möglichen Freizeitformen).

In Anlehnung an das schweizerische Tourismuskonzept läßt sich gedanklich die Fremdenverkehrsforschung wohl in die drei Teilbereiche Gesellschaft, Wirtschaft und Umwelt aufteilen; da indessen Fremdenverkehr eine typische Querschnittswissenschaft ist und das Augenmerk vermehrt von der sektoriellen Betrachtung auf die Gesamtzusammenhänge ausgedehnt werden muß, betreibt das FIF heute hauptsächlich interdisziplinäre Grundlagenforschung in Zusammenarbeit mit Vertretern anderer Wissenschaften.

\section{Lehre}

Fremdenverkehr ist seit jeher ein Wahlfach im Rahmen des rechts- und wirtschaftswissenschaftlichen Studiums an der Universität Bern. Da die maximale Stundenzahl der Fremdenverkehrsveranstaltungen einen Achtel des Gesamtstudiums nicht überschreiten darf, ist eine eigentliche Tourismus-Spezialisierung im Gegensatz zu einigen ausländischen Fachhochschulen - an der Universität Bern weder möglich noch angestrebt.

$\mathrm{Zu} \mathrm{Beginn} \mathrm{der} \mathrm{Institutstätigkeit} \mathrm{wurde} \mathrm{wöchentlich}$ eine zweistündige Vorlesung angeboten, wobei sich die Auswahl während mehrerer Jahre im Winter auf die "Grundzüge der Fremdenverkehrslehre» und im

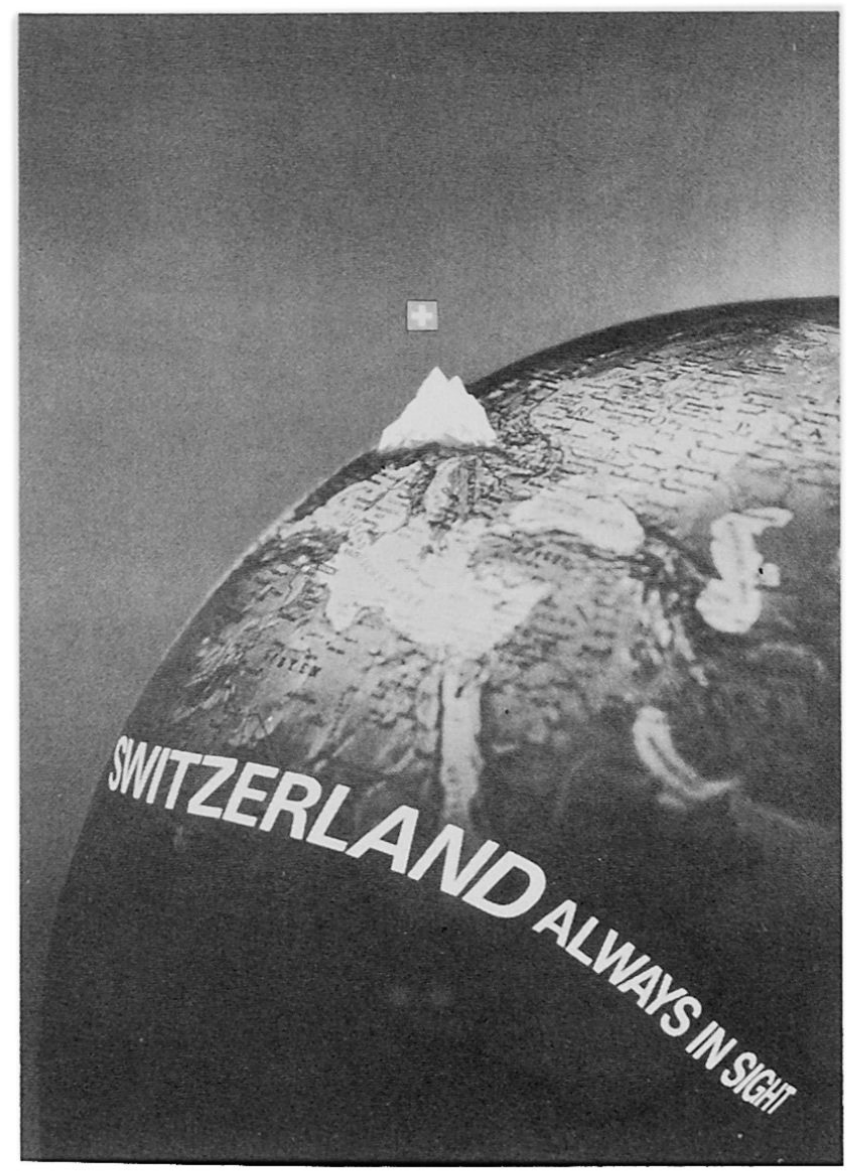

Beispiel eines Werbeplakats der Schweizerischen Verkehrszentrale

Sommer auf die «Fremdenverkehrspolitik» beschränkte. Prof. Krapf erkannte indessen schon früh, $\mathrm{da} ß$ ein steter Bezug zur Praxis unabdingbare Voraussetzung für eine erfolgreiche Tätigkeit des FIF war: So veranstaltete er 1944 erstmals eine (eintägige) Fachexkursion in ein Fremdenverkehrsgebiet, um den Studenten Gelegenheit zu praktischem Anschauungsunterricht und Kontakt zu den Tourismusverantwortlichen zu bieten. Heute gehören alljährliche Fachexkursionen (mehrtägig, zum Teil ins Ausland) gewissermaßen zur Tradition des FIF. Ferner führte Prof. Krapf 1945 ein wöchentlich einstündiges Kolloquium zu aktuellen Fragen des Fremdenverkehrs ein, um neben dem Vorlesungsstoff ausgewählte Probleme mit den Studenten und eingeladenen Fremdenverkehrspraktikern diskutieren zu können.

Im Gefolge der ausgeweiteten Forschungstätigkeit änderten sich auch Inhalt und Zyklus der Vorlesungen. Heute werden den Studenten wöchentlich drei Vorlesungsstunden (mit integriertem Kolloquium) zu folgenden Semester-Themen angeboten:

- Fremdenverkehr und Wirtschaft I (Fremdenverkehrslehre und -politik),

- Fremdenverkehr und Wirtschaft II (Marketing im Fremdenverkehr), 
- Fremdenverkehr und Gesellschaft (insbesondere Fremdenverkehr in Entwicklungsländern),

- Fremdenverkehr und Umwelt (insbesondere raumplanerische Aspekte des Fremdenverkehrs).

Besuchten am Anfang zwischen 25 und 30 Studenten die einzelnen Veranstaltungen, so hat sich diese Zahl bis heute mehr als verdoppelt. Grundlegend geändert hat sich auch die Vorlesungsgestaltung: Waren bis Ende der siebziger Jahre sogenannte Magistralvorlesungen die Regel, so wird heute ein aktiver Einbezug der Studenten gesucht: Das Basiswissen wird zu Beginn des Semesters schriftlich (in Buchform) abgegeben; während des Semesters werden dann ausgewählte (aktuelle) Probleme durch Beizug von Praktikern mit den Studenten diskutiert und Lösungsansätze erarbeitet. Wenn immer möglich werden diese Veranstaltungen durch audiovisuelle Hilfsmittel unterstützt (Video, Film, Dias, Besichtigungen u. ä.).

\section{Beratung/Gutachten}

Die betriebswirtschaftliche Orientierung schlug sich zu Beginn auch in der Beratungs-/Gutachtertätigkeit nieder. Es ist in diesem Zusammenhang interessant zu wissen, daß das FIF bis 1968 eine spezielle betriebswirtschaftliche "Abteilung» unterhielt. In den sechziger und siebziger Jahren wirkte das Institut dann namentlich als touristischer Fachberater bei der Erarbeitung regionaler Entwicklungskonzepte im (bernischen) Berggebiet. Ferner wurden häufig sogenannte «Junior-Experten» in Entwicklungsländern eingesetzt und Stipendiaten aus dem Ausland am FIF betreut. In jüngster Zeit standen marketingpolitische Aufträge im Vordergrund. Als Beispiele seien genannt: «Leitbild und Verbandspolitik des Schweizer Hotelier-Vereins» sowie "Touristisches Marketing im Kanton Bern mit Schwerpunkt Marktbearbeitung». Ferner beteiligt sich das Institut nach wie vor aktiv an der touristischen Aus- und Weiterbildung von Fremdenverkehrspraktikern (insbesondere Seminar «Unternehmungsführung in Hotellerie und Gastgewerbe» des Schweizer Hotelier-Vereins).

\section{Dokumentation}

Seit seiner Gründung betreut das FIF eine öffentlich zugängliche Bibliothek zu allen einschlägigen Fragen des Fremdenverkehrs. Verfügte das Institut beispielsweise 1943 erst über 180 Bücher und einige Zeitschriften, so stehen dem Besucher heute in Freihandaufstellung rund 3900 Bücher (davon 1500 vor 1968 erschienene Werke in einem getrennten Archiv) und etwa 90 Periodika zur Verfügung. Am Anfang wurden die Bücher noch ausgeliehen (teilweise bis 400 Ausleihungen pro Jahr); angesichts mehrerer Verluste und zahlreicher unersetzbarer Einzelexemplare wurde die Bibliothek im Zuge einer umfassenden Neugestaltung 1978 als reiner Präsenzbestand (also ohne Ausleihmöglichkeit) aufgebaut. Sie steht jedoch nach wie vor jedermann (nicht nur Institutsmitarbeitern und Studenten) unentgeltlich zur Verfügung und wird entsprechend rege benützt. Ebenfalls sehr geschätzt wird eine weitere kostenlose Dienstleistung des FIF: Interessenten können sich zu genau umschriebenen Themen Bibliographien verfügbarer Werke ausfertigen lassen. Nach Möglichkeit werden solchen Anfragen auch immer einige aktuelle Zeitungsausschnitte zum interessierenden Problembereich beigelegt.

\section{Publikationen}

Zum Aufgabenbereich des FIF gehört schließlich implizit die Weiterleitung der Forschungsergebnisse an die Praxis. Neben Vorträgen und regelmäßigen Artikeln in Zeitungen und Zeitschriften geschieht dies insbesondere auf vier Wegen: Einmal gibt das FIF die institutseigene Schriftenreihe «Berner Studien zum Fremdenverkehr» heraus. Diese umfaßt zur Zeit 19 Bände. Darin werden besonders aufschlußreiche und wertvolle Studien eher grundsätzlicher Art veröffentlicht. Als Begleitlektüre zum Fremdenverkehrsstudium - aber auch als Nachschlage- und Informationswerk für die Praxis - besteht daneben die Reihe «Dokumentationen zum Fremdenverkehrsstudium» (Bestand zur Zeit 4 Hefte). In den Jahresberichten über die FIF-Tätigkeit erscheint jeweils ein Fachbeitrag, und schließlich gibt das Institut zusammen mit jenem der Hochschule St. Gallen die viermal jährlich erscheinende "Zeitschrift für Fremdenverkehr» (Revue de Tourisme) heraus.

Forschungsinstitut für Fremdenverkehr Monbijoustr. 29, 3011 Bern 